PROCEDURES AND BASIC STATISTICS TO BE USED IN MAGNITUDE CONTROL OF EQUALISATION RESERVES IN FINLAND

\title{
Esa Hovinen \\ Helsinki
}

This report aims at describing the procedures and statistics prepared for use in calculation of the limits of the equalisation reserves of Finnish insurance companies. The report is based on work done by a committee which the Federation of Finnish Insurance Companies set up in I962. Its purpose was to collect and work up the necessary statistics and to develop computational methods to be used in practice for the computation of equalisation reserves. In one respect the work was initiated by the Supervisory Service, which prepared new and more precise regulations concerning the determination of limits of the above reserves.

Because solvency problems of insurance companies have had great attention in recent years internationally, and the Finnish equalisation reserves are closely related to them, the theme may be sufficiently interesting to be presented once more setting out practical results. The solvency problem among other questions has been dealt with by Drs. Pesonen and Pentikäinen in former colloquia. This report can be considered as a continuation of Dr. Pesonen's paper "Magnitude Control of Technical Reserves in Finland" submitted to the Lucerne Colloquium and my paper "A Procedure to Compute Values of the Generalised Poisson Function" to the same colloquium.

\section{Principles and practical formulae}

The principles and theoretical formulae to be used in computation of the limits for the equalisation reserves have already been stated in the papers of Drs. Pesonen and Pentikäinen. Other technical reserves are not dealt with here.

The equalisation reserve is roughly defined as that part of the technical reserves which exceeds the conventional premium reserve 
and the claims reserve and which secures the company's solvability against random fluctuations of claims and fluctuation of the basic probabilities of the claims. The equalisation reserve $E$ must be greater than a minimum $E_{\min }$ from solvability reasons and, because of taxation, should not exceed a maximum amount $E_{\max }$.

Let us denote by

$x$ the total amount of claims during one year on a company's own risk,

$P$ the corresponding net premium income,

$U$ the company's own capital and free reserves,

$\lambda$ the security margin in premiums and

$i$ the rate of interest.

If the company's size and the structure of its portfolio are assumed to remain the same, the equation

$$
\operatorname{Pr}\left\{(\mathrm{I}+i)\left(E_{\min }+U\right)+\sqrt{\mathrm{I}+\mathrm{i}}[(\mathrm{I}+\lambda) P-x] \geq 0\right\}=\mathrm{I}-\varepsilon
$$

can serve as a definition of the minimum amount $E_{\mathrm{min}}$. If the reserve $E=E_{\mathrm{min}}$, the probability of ruin after one year is $\varepsilon .{ }^{1}$ )

The total amount of claims $x$ is assumed to have the distribution

where

$$
F(x)=\sum_{r=0}^{\infty} p_{\bar{n}}(r) S^{*}(x)
$$

$\bar{n}=$ the expected number of claims $=\Sigma \bar{n}_{k}$,

$p_{\bar{n}}(r)=$ the probability of $r$ claims occurring and

$S(x)=\sum_{k} \frac{\bar{n}_{k}}{\bar{n}} S_{k}(x)$, where $S_{k}(x)$ is the distribution of one claim

in branch $k$. The reinsurer's share is excluded except as regards Stop Loss treaties, which are taken into account in a later phase of the calculations.

The number of claims in the following is assumed to have the Poisson distribution

$$
p_{\hat{n}}(r)=e^{-\bar{n}} \frac{\bar{n}^{r}}{r !}
$$

1) A further restriction posed on $E_{\min }$ in Finland is

$$
E_{\min } \geq \operatorname{Max}\{0, M-U\} \text {, }
$$

where $M$ is the company's maximum net retention defined realistically. 
A Polya distribution would also have been possible, with the following methods valid mutatis mutandis.

According to the instructions of the Supervisory Service in Finland, the fluctuation of the basic probabilities of the claims is taken into account by taking

$$
\bar{n}_{k}=\left(\mathrm{I}+q_{k}\right) n_{k}
$$

where the number $q_{k}$ is a constant estimated from experience in branch $k$ and $n_{k}$ the estimated number of claims.

Let us denote

$$
\alpha_{k i}^{\prime}=\int_{0}^{\infty} x^{i} d S_{k}(x)
$$

and

$$
\alpha_{k i}=\frac{\alpha_{k i}}{\left(\alpha_{k 1}\right)^{i}}
$$

For the distribution (2) we then have

$$
\begin{aligned}
E\{x\} & =\Sigma \bar{n}_{k} \alpha_{k 1}=\Sigma \bar{P}_{k}=\Sigma\left(\mathrm{x}+q_{k}\right) P_{k} \\
\sigma^{2}\{x\} & =\mu_{2}=\Sigma \bar{n}_{k} \alpha_{k 2}=\sum \frac{\bar{P}_{k}^{2}}{\bar{n}_{k}} \alpha^{\prime}{ }_{k 2} \\
\mu_{3} & =\Sigma \bar{n}_{k} \alpha_{k 3}=\sum \frac{\bar{P}_{k}^{3}}{\bar{n}_{k}^{2}} \alpha_{k 3 .}^{\prime} .
\end{aligned}
$$

If

$$
\frac{\mu_{3}}{\sigma^{3}}<2.5,
$$

which is always the case if

$$
M<2.5 \sigma,
$$

then, according to Kauppi and Ojantakanen (Approximations of the generalised Poisson function, ASTIN-Colloquium in Arnhem), the value of $x_{\varepsilon}$ for which $F\left(x_{\varepsilon}\right)=\mathrm{I}-\varepsilon$, is, with a reasonable accuracy,

$$
x_{\varepsilon} \approx \Sigma \bar{P}_{k}+y_{\varepsilon} \cdot \sigma+\frac{\mathrm{I}}{6} \frac{\mu_{3}}{\sigma^{2}}\left(y_{\varepsilon}^{2}-\mathrm{I}\right),
$$


where $y_{\varepsilon}$ is the root of the equation $\phi\left(y_{\varepsilon}\right)=I-\varepsilon$. The expression (4) holds true for almost all companies.

After a little manipulation we find from equations (I) and (5)

$E_{\min }=\frac{I}{\sqrt{I}+i}\left\{\Sigma\left(q_{k}-\lambda\right) P_{k}+y_{\varepsilon} \cdot \sigma+\frac{I}{6} \frac{\mu_{3}}{\sigma^{2}}\left(y_{\varepsilon}^{2}-\mathrm{I}\right)\right\}-U$.

Inserting the values $i=0.05, \lambda=0, \varepsilon=0.0 \mathrm{I}$ as posed by the Supervisory Service gives

$$
E_{\min }=0.976 \Sigma q_{k} P_{k}+2.270 . \sigma+0.7 \mathrm{I} 4 \frac{\mu_{3}}{\sigma^{2}}-U .
$$

The maximum amount $\mathrm{E}_{\max }$ can, in principle, be defined by

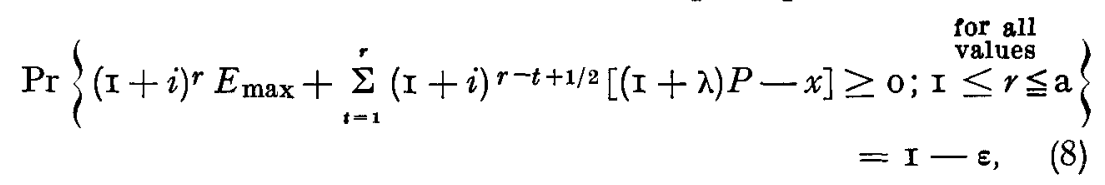

if the company is allowed to retain the amount $U$.

In Finland, we also have the further restriction

$$
E_{\max } \geq 2 M \text {. }
$$

For the values $i=0.05, \lambda=0, a=5$ and $\varepsilon=0.0 \mathrm{I}$ it has been noticed that the equation

$$
\operatorname{Pr}\left\{1.05^{5} E_{\max }+\sum_{t=1}^{5}(\mathrm{I} .05)^{5-t+1 / 2}(P-x) \geq 0\right\}=0.99
$$

gives the same $E_{\max }$ as equation (7) with an accuracy wholly sufficient for insurance companies. Applying equation (5) we get after a little manipulation

$$
E_{\max }=4.436 \Sigma q_{k} P_{k}+4.626 . \sigma+0.658 \cdot \frac{\mu_{3}}{\sigma^{2}} .
$$

\section{Statistics and tables for use}

The committee has collected extensive statistics to obtain the functions $S_{k}(x)$, the records of large claims extending over many years being brought into the experience. The resulting functions $S_{k}(x)$ are presented as graphs in Appendix I. Moreover the tails of the distributions $S_{k}(x)$ are smoothed and continued according 
to a Pareto-distribution for a decade longer than they were in the empirical material. The influence of inflation has naturally been eliminated.

The functions $S_{k}(x)$ relate to the gross claims. The insurance companies have, however, various reinsurance contracts which must be taken into account. Therefore, working tables were computed, where the characteristics

$$
\begin{aligned}
& M_{k}^{\prime}=\frac{M_{k}^{*}}{\alpha_{k 1}^{*}} \\
& M_{k}^{*} \\
& \alpha_{k 1}^{*}=\int_{0}^{M_{k}^{*}} x d S_{M_{k}^{*}}(x) \\
& \alpha_{k 2}^{\prime}=\frac{\alpha_{k 2}^{*}}{\left(\alpha_{k 1}^{*}\right)^{2}} \\
& \alpha_{k 3}^{\prime}=\frac{\alpha_{k 3}^{*}}{\left(\alpha_{k 1}^{*}\right)^{3}}
\end{aligned}
$$

of the distributions

$$
S_{M_{k}^{*}}(x)=\left\{\begin{array}{cl}
S_{k}(x) & x<M_{k}^{*} \\
\mathrm{I} & x \geq M_{k}^{*}
\end{array}\right.
$$

are represented. Three examples of such tables are presented in Appendix 2. The second and third columns in these are not needed in standard computations.

The tables are used in the following way. First, the insurances in force are divided into branches (classes) $k$. Then, the net premium incomes $P_{k}$ and the expected numbers of claims $n_{k}$ are determined according to special instructions. The third task is to determine realistically the maximum net retention $M_{k}$ in branch $k$. Then

and

$$
\alpha_{k 1}=\frac{P_{k}}{n_{k}}
$$

$$
M_{k}^{\prime}=\frac{M_{k}}{\alpha_{k 1}}
$$


The numbers $\alpha_{k 2}^{\prime}$ and $\alpha_{k 3}^{\prime}$ are derived by interpolation from the working tables and formulae (7) and (ro) applied.

The above method automatically takes into account the effect of inflation and quota share reinsurance. It has also been considered a sufficient way of taking the various reinsurance methods (excess of loss, excedent, mixed methods) into account.

\section{Some exceptions}

One exception to the above is Stop Loss reinsurance, which has its own rules.

Certain branches have no working table. They are divided into relatively homogeneous classes $i$, and then $P_{k i}$ and $M_{k i}$ determined. The number $n_{k i}$ is defined as

$$
n_{k i}=\frac{P_{k i}}{M_{k i}}
$$

after which the numbers

$$
\alpha_{k 2 i}^{\prime}=\mathbf{I}
$$

and

$$
\alpha_{k 3 i}^{\prime}=\mathrm{I}
$$

are used. The method is based on Pesonen's paper "On the calculation of the generalised Poisson function", par. 7, 5th AstinColloquium.

There are some situations in which the above mentioned methods are inapplicable. Then case by case estimation methods and perhaps Monte Carlo methods and computers are used. Prepared computer programs also make it possible to deal with exceptional situations.

\section{Conclusions}

I have tried to give a picture of the rules and statistics intended as standard in computing limits for the equalisation reserves in Finnish insurance companies. These reserves are compulsory and intended to secure the companies' solvability in case of exceptional high total losses.

The rules and statistics seem to meet the problem well. They are quite simple and easy to use in all our companies, even if required 
every year. Yet they seem to be nearly as accurate as it is possible in practice, even for parameter values other than those posed by the Finnish Supervisory Service.

\section{Appendix I}

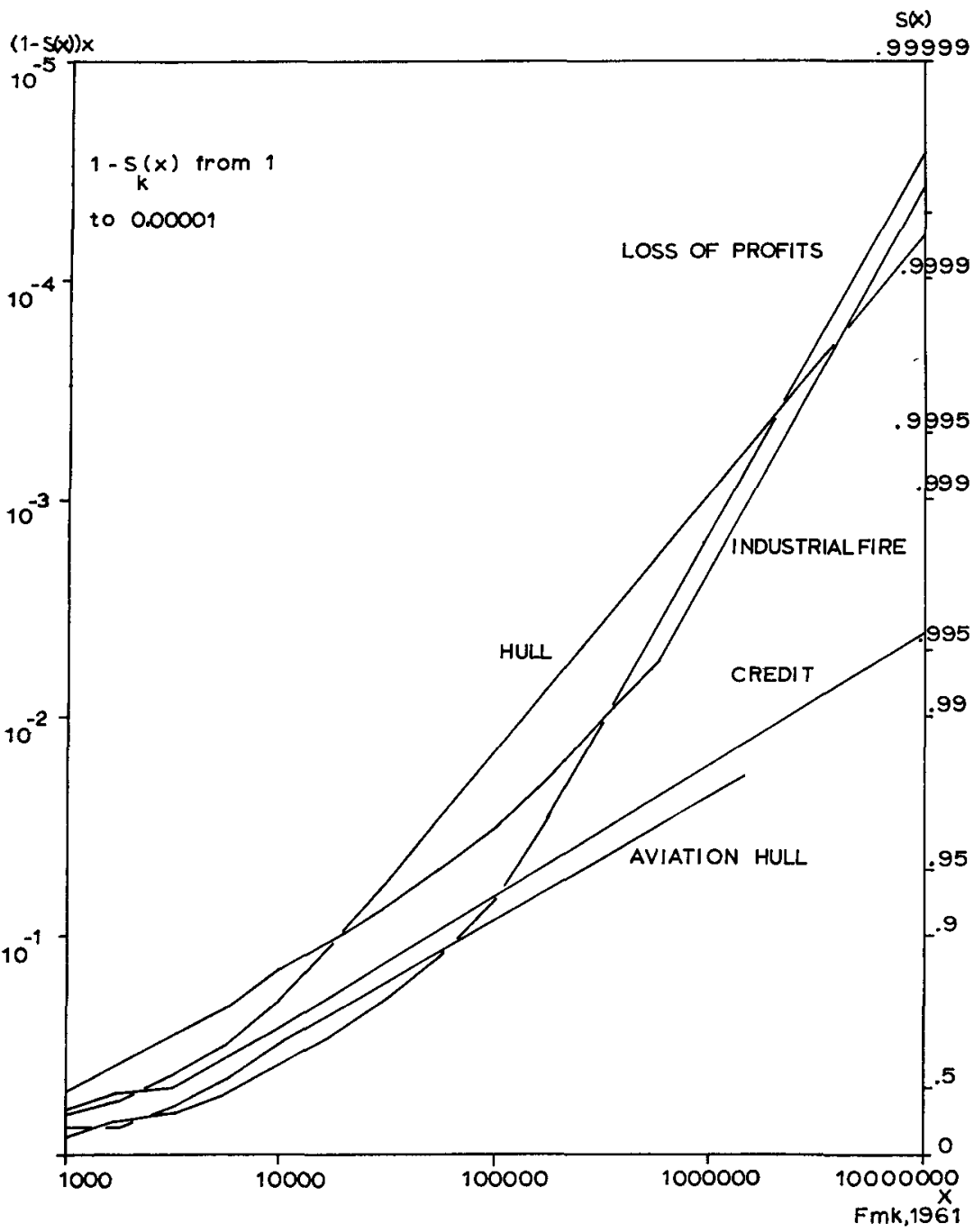

Fig. I. 


\section{Appendix I}

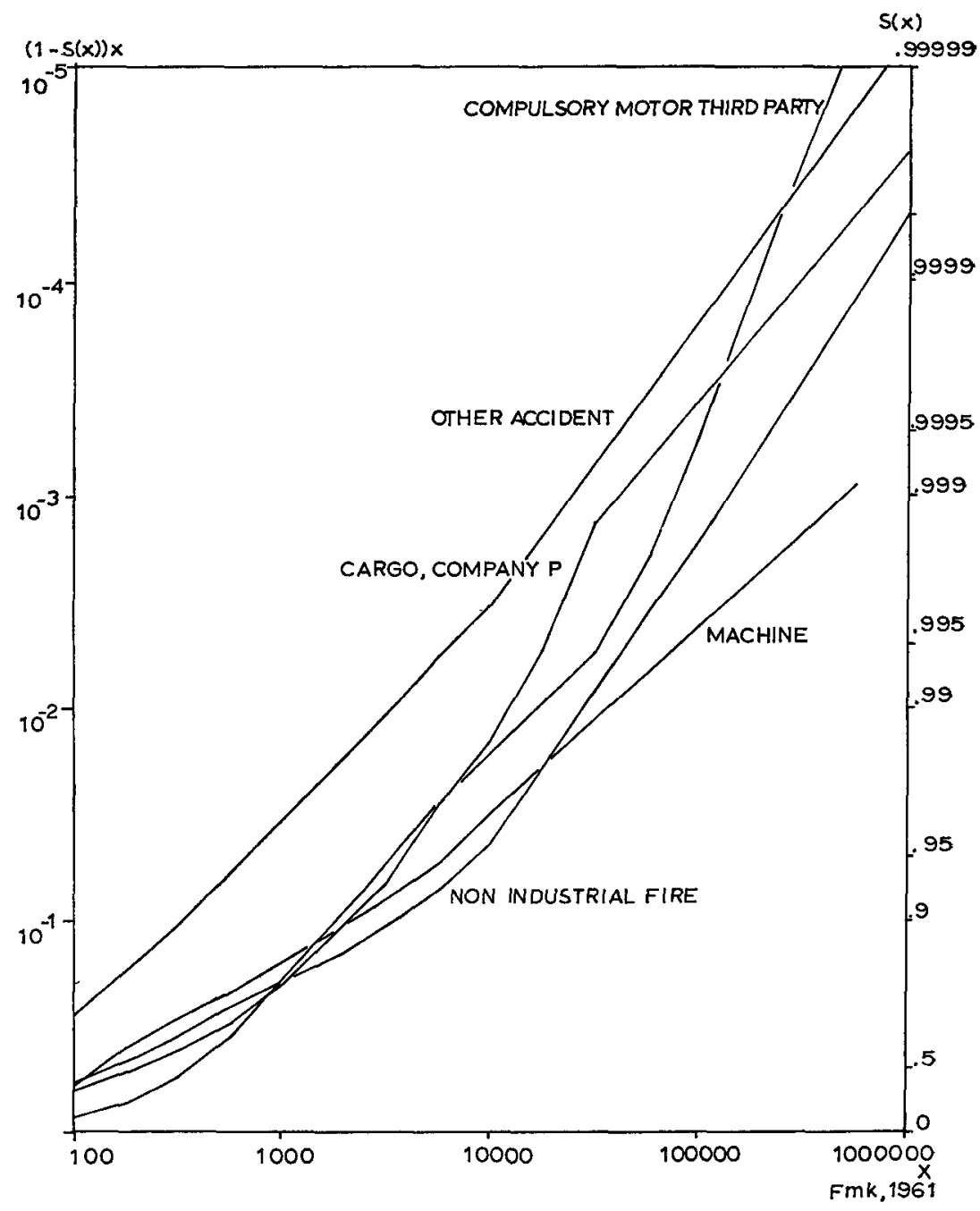

Fig. 2. 
Appendix I

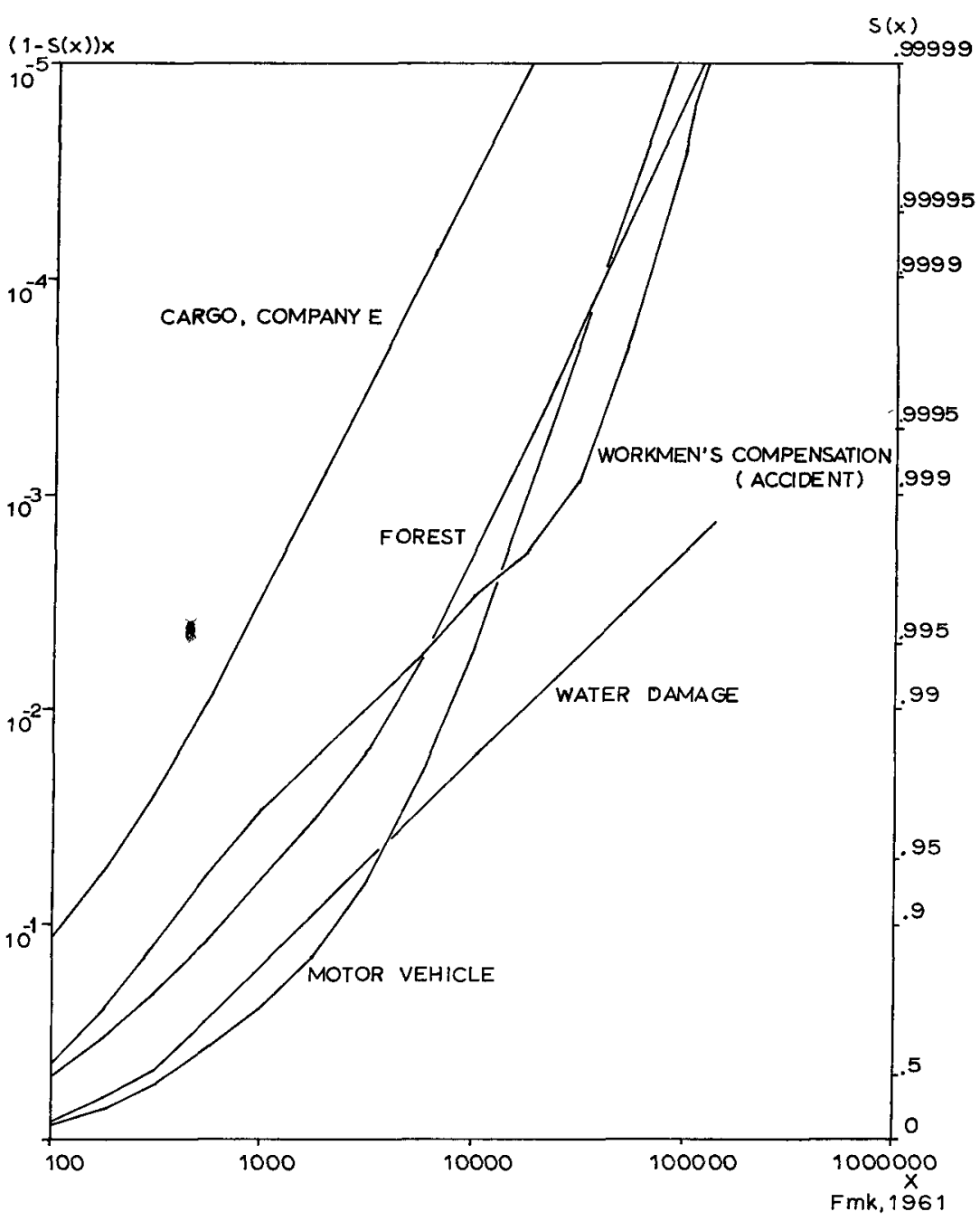

Fig. 3 . 
Appendix I

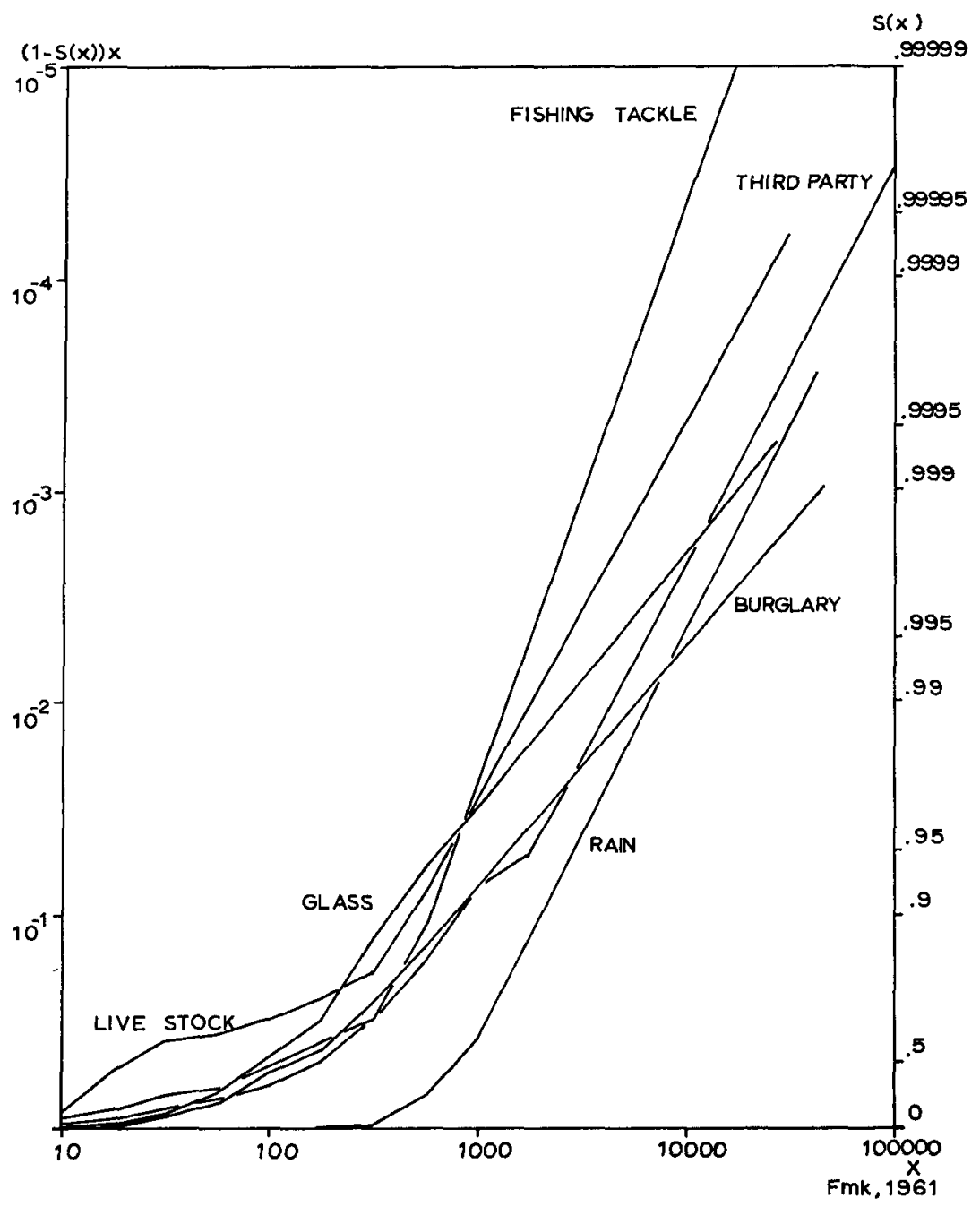

Fig. 4. 
APPENDIX 2

\section{EXAMPLES OF WORKING TABLES}

\section{Credit insurance}

\begin{tabular}{rr}
$M^{\prime}{ }_{k}$ & \multicolumn{1}{c}{$M^{*}{ }_{k}$} \\
& Fmk, I96r \\
1,7 & 2512 \\
1,8 & 3981 \\
2,0 & 6310 \\
2,3 & 10000 \\
2,8 & 15850 \\
3,5 & 25120 \\
4,4 & 39810 \\
5,5 & 63100 \\
7,0 & 100000 \\
9,0 & 158500 \\
11,6 & 251200 \\
15,1 & 398100 \\
19,6 & 631000 \\
25,6 & 1000000 \\
33,4 & 1585000 \\
43,7 & 2512000 \\
57,3 & 3981000 \\
75,2 & 6310000 \\
98,7 & 10000000 \\
129,8 & 15850000 \\
170,7 & 25120000 \\
224,6 & 39810000
\end{tabular}

$\alpha^{*} k 1$
Fmk, I96
I477
2213
3152
4279
5621
7223
9147
11459
14235
17570
21574
26384
32164
39103
47441
57453
69478
83926
101275
122119
I47150
I7721

$\alpha_{k 2}^{\prime}$

$\alpha_{k 3}^{\prime}$

512

$398 \mathrm{I}$

6310

10000

25120

39810

63100

158500

251200

398 100

631000

I000000

1585000

2512000

3981000

6310000

rooooooo

25120000

*1

1477

2213

3152

4279

5621

7223

9147

11459

14235

7570

21574

26384

39103

$4744 \mathrm{I}$

57453

$6947^{8}$

83926

OI 275

47150

$772 \mathrm{II}$

$\mathbf{I}, 6$

1,7

$\mathrm{I}, 8$

$2,0 \quad 4$

$2,2 \quad 5$

$2,6 \quad 7$

$3, \mathrm{I}$ II

$3,8 \quad 18$

$4,6 \quad 28$

$5,8 \quad 46$

$7,3 \quad 75$

$9,3 \quad 123$

I $2,0 \quad 205$

I5,4 345

$20,0 \quad 583$

$25,9 \quad 990$

$33,7 \quad I 689$

$44,0 \quad 2891$

$57,5 \quad 4963$

$75,3 \quad 8542$

$98,7 \quad$ I $473^{\circ}$

$129,5 \quad 2544^{\circ}$

\section{Industrial fire insurance}

\begin{tabular}{rr}
$M^{\prime}{ }_{k}$ & \multicolumn{1}{c}{$M^{*}{ }_{k}$} \\
& Fmk, I96I \\
I,6 & I4I3 \\
1,8 & 2239 \\
2,2 & $354^{8}$ \\
2,7 & 5623 \\
3,3 & 8913 \\
$4, \mathrm{I}$ & I4I30 \\
5,2 & 22390 \\
6,7 & 35480 \\
8,6 & 56230 \\
$\mathrm{II,3}$ & 89130 \\
$\mathrm{I} 5,0$ & I4I300 \\
20,4 & 223900 \\
28,3 & 354800 \\
40,1 & 562300 \\
58,5 & 891300
\end{tabular}

$\alpha^{*} k 1$

$\alpha^{\prime}{ }_{k 2}$

$\alpha^{\prime}{ }_{k 3}$

Fmk, I96I

893
1212
1618
2118
2703
3406
4273
5307
6510
7897
9433
10991
12529
14024
15244

$\mathbf{I}, 4$

I,5

$\mathrm{r}, 7$

2,0

2,4

2,9

3,6

4,4

5,5

6,9

8,8

II, I

I 4,1

18,1

22,7

2
2
3
4
6
10
16
25
41
67
111
185
318
561
956


238 CONTROL OF EQUALISATION RESERVES IN FINLAND

\begin{tabular}{|c|c|c|c|c|}
\hline \multirow[t]{2}{*}{$M^{\prime}{ }_{k}$} & $M^{*}{ }_{k}$ & $\alpha^{*} k 1$ & $\alpha_{k 2}^{\prime}$ & $\alpha_{k 3}^{\prime}$ \\
\hline & Fmk, I96I & Fmk, I96I & & \\
\hline 87,5 & $\mathrm{I}_{4} \mathrm{I}_{3} 3000$ & I6I 41 & 27,9 & I6I 8 \\
\hline I 33,4 & 2239000 & I 6778 & 33,9 & $273^{\mathrm{I}}$ \\
\hline 205,9 & 3548000 & $\mathbf{r} 7233$ & 40,7 & $465^{\circ}$ \\
\hline 320,3 & 5623000 & I 7556 & 48,5 & 7999 \\
\hline $5 \circ 1,1$ & 8913000 & I 7786 & 57,5 & I 3882 \\
\hline 787,2 & $I_{4} I_{3} 0000$ & I $795^{\circ}$ & 67,8 & 24293 \\
\hline I 239,4 & 22390000 & I 8065 & 79.5 & $4260 I$ \\
\hline I955,0 & 35480000 & $18 I_{4} 8$ & 92,9 & 75299 \\
\hline 3088,4 & 56230000 & r 8207 & I08, I & I 33292 \\
\hline $4884, \mathrm{I}$ & 89130000 & I 8249 & I 25,4 & $23727^{\circ}$ \\
\hline 7729,3 & I 4 I 300000 & I $828 \mathrm{I}$ & I 46,0 & 432189 \\
\hline
\end{tabular}

Compulsory motor third party insurance

\begin{tabular}{|c|c|c|c|c|}
\hline$M_{k}^{\prime}$ & $\begin{array}{c}M^{*}{ }_{k} \\
\text { Fmk, I96I }\end{array}$ & $\begin{array}{c}\alpha^{*}{ }_{k 1} \\
\text { Fmk, I } 1961\end{array}$ & $\alpha_{k 2}^{\prime}$ & $\alpha_{k 3}^{\prime}$ \\
\hline 1,8 & 508 & 400 & $I, 4$ & 2 \\
\hline 2,3 & I 122 & 494 & $I, 6$ & 3 \\
\hline 3,0 & I 778 & 583 & 1,9 & 4 \\
\hline 4,2 & 2818 & 665 & 2,3 & 7 \\
\hline 6,0 & 4467 & $74 \mathrm{I}$ & 2,8 & 12 \\
\hline 8,7 & 7080 & 815 & 3,6 & 22 \\
\hline 12,6 & II 220 & 890 & 4,7 & 43 \\
\hline I 8,4 & $\mathrm{I} 7780$ & 968 & 6,4 & 87 \\
\hline 26,8 & 28180 & ro5o & 8,8 & I 78 \\
\hline 39,7 & $44^{6} 7^{\circ}$ & I 126 & I I,9 & 349 \\
\hline 60,1 & 70800 & $\operatorname{II} 78$ & I $5, \mathrm{I}$ & 609 \\
\hline 92,9 & I I 2200 & 1208 & $x 7,9$ & 962 \\
\hline $\mathrm{I} 45,5$ & I77800 & 1222 & 20,1 & I 372 \\
\hline 229,3 & $28 \mathrm{I} 800$ & 1229 & $2 \mathrm{I}, 8$ & $\times 892$ \\
\hline 362,6 & 446700 & 1232 & 23,3 & 2547 \\
\hline 573,7 & 708000 & I 234 & 24,4 & 3364 \\
\hline 908,5 & I I 22000 & 1235 & 25,3 & $43^{88}$ \\
\hline 439,7 & I 778000 & I235 & $26, \mathrm{I}$ & $567 \mathrm{I}$ \\
\hline 2279,9 & 2818000 & 1236 & 26,6 & 7164 \\
\hline
\end{tabular}

